\title{
Influence of NOD2 Variants on Trichuris suis ova Treatment Outcome in Crohn's Disease
}

\section{OPEN ACCESS}

Edited by:

Raffaele Capasso,

Università degli Studi di Napoli

Federico II, Italy

Reviewed by:

Matteo Fornai,

Università degli Studi di Pisa, Italy Irina Leonardi,

Cornell University, United States

*Correspondence:

Simon U. Jaeger

simon.jaeger@ikp-stuttgart.de

Jan Wehkamp

jan.wehkamp@med.uni-tuebingen.de

Specialty section:

This article was submitted to Gastrointestinal and Hepatic

Pharmacology,

a section of the journal

Frontiers in Pharmacology

Received: 18 April 2018

Accepted: 22 June 2018

Published: 16 July 2018

Citation:

Jaeger SU, Schaeffeler E, Winter S,

Tremmel R, Schölmerich J, Malek N,

Stange EF, Schwab M and

Wehkamp J (2018) Influence of NOD2

Variants on Trichuris suis ova

Treatment Outcome in Crohn's

Disease. Front. Pharmacol. 9:764.

doi: 10.3389/fphar.2018.00764

\section{Simon U. Jaeger ${ }^{1,2 *}$, Elke Schaeffeler ${ }^{1,2}$, Stefan Winter ${ }^{1,2}$, Roman Tremmel ${ }^{1,2}$, Jürgen Schölmerich ${ }^{3}$, Nisar Malek ${ }^{4}$, Eduard F. Stange ${ }^{4}$, Matthias Schwab ${ }^{1,2,5,6}$ and Jan Wehkamp ${ }^{* *}$}

${ }^{1}$ Dr. Margarete Fischer Bosch Institute of Clinical Pharmacology, Stuttgart, Germany, ${ }^{2}$ University of Tübingen, Tübingen, Germany, ${ }^{3}$ Goethe University Frankfurt, Frankfurt, Germany, ${ }^{4}$ Department of Internal Medicine I, University of Tübingen, Tübingen, Germany, ${ }^{5}$ Department of Clinical Pharmacology, University of Tübingen, Tübingen, Germany, ${ }^{6}$ Department of Pharmacy and Biochemistry, University of Tübingen, Tübingen, Germany

A recent randomized study of whipworm Trichuris suis ova (TSO) in ileal Crohn's disease failed to demonstrate a clinical benefit compared to placebo after 12 weeks. Nonetheless, it has recently been shown that the spontaneous small intestinal inflammatory changes in Nod2- / - (Nucleotide-binding oligomerization domain 2) mice could be substantially ameliorated when these mice were colonized by Trichuris muris. Those and complementary epidemiologic findings in humans lead to the hypothesis that helminths may be advantageous only in patients carrying defective NOD2 variants. Thus, 207 participants of the TSO trial were retrospectively genotyped for six functional NOD2 genetic variants to evaluate whether the treatment outcome differed in patients carrying NOD2 variants. We observed no significant association of the NOD2 variants or their haplotypes with clinical outcome after TSO treatment.

Keywords: Trichuris suis ova, Crohn's disease, NOD2, helminths, innate immunity

\section{INTRODUCTION}

Inflammatory bowel disease is most common in the Western world where helminthic colonization is rare. Along the lines of the so-called hygiene hypothesis, the presence of helminths including whipworms like Trichuris trichiura can be inversely correlated with the manifestation of immunerelated conditions like Crohn's disease. Yet, epidemiologic influences are difficult to entangle and possible mechanisms are still poorly understood.

The first indications for therapeutic efficacy of a helminth-based therapy came from two unblinded clinical trials which investigated viable Trichuris suis ova (TSO) both in Crohn's disease and ulcerative colitis (Summers et al., 2005a,b). A study with a single administration of embryonated, viable ova of Trichuris suis, up to 7500 eggs, showed a good tolerability (Sandborn et al., 2013) but was not designed to evaluate response. This was addressed in the recent trial by Schölmerich et al. (2017), in which the clinical efficacy of a fortnightly suspension of three doses of TSO in mildly to moderately active, uncomplicated Crohn's disease over 12 weeks were tested against placebo. In contrast to the previous studies, this first double-blind and randomized trial failed to demonstrate a clinical benefit for TSO compared to placebo, at least after 12 weeks of treatment.

The failure of the study might be explained by an insufficient therapy duration or by the unusually high placebo remission rate (42.9\%). In addition, the authors considered that the 
prevention of disease relapse might have been a more suitable endpoint. Yet, it has to be acknowledged that the interplay between host immune function and Trichuris species is complex and remains insufficiently understood. Helminth products are known to modulate the host immune response, as for example, E/S glycans of the whipworm T. suis suppress LPS-induced cytokine production by intestinal epithelial cells and by dendritic cells. Furthermore, mucus production is increased (Hiemstra et al., 2014), which may facilitate helminth expulsion.

A role for the cytosolic pattern recognition receptor Nod2 (Nucleotide-binding oligomerization domain 2) in the host response to Trichuris species (in this case Trichuris muris) has been demonstrated in colonic epithelial cells in Nod2 $2^{-/-}$mice. In the absence of Nod2, dendritic cell recruitment is impaired, which leads to an increased worm burden at day 21 alongside lower numbers of $\mathrm{T}$ cells in mesenteric lymph nodes (Bowcutt et al., 2014).

It has also been shown that $\mathrm{Nod} 2^{-/-}$mice harbor several small intestinal abnormalities including increased proportion of IFN- $\gamma$ expressing intraepithelial lymphocytes, goblet cell defects, and altered antimicrobial peptide expression. Nod $2^{-/-}$mice had a significantly different microbiome and the small intestinal changes depend on expansion of the Gram-negative anaerobic coccobacillus Bacteroides vulgatus. Accordingly, Nod2 prevents inflammation by restricting the pathological expansion of this important commensal bacterium (Ramanan et al., 2014).

In the recent study "Helminth infection promotes colonization resistance via type 2 immunity" by Ramanan et al. (2016), Nod $2^{-/-}$mice were infected with $\sim 25$ embryonated T. muris ova and evaluated at day 35 post infection. The small intestinal changes which arise in $N o d 2^{-/}$mice could be substantially ameliorated when these mice were colonized by T. muris. The helminth colonization induced a type 2 immunity response that favored the expansion of Clostridiales, which in turn inhibit colonization with the gram-negative anaerobic coccobacillus B. vulgatus and other Bacteroidales.

It was hypothesized from these findings and further epidemiologic evidence in humans that helminths may be beneficial only in patients with NOD2 variants or proinflammatory Bacteroides species (Ramanan et al., 2016). The study design of the original TSO trial did not incorporate NOD2 genetics, and thus it seemed worthwhile to investigate if outcome would in fact differ in those patients carrying genetic variants of NOD2.

\section{MATERIALS AND METHODS}

\section{Study Population}

The study population is a subgroup from the abovementioned clinical trial (Schölmerich et al., 2017). Genetic analyses could be conducted in 207 patients who had signed an additional written informed consent at the beginning of the clinical trial, i.e., 60 patients treated with placebo, and 32,62 , and 53 patients treated with 250, 2500, and 7500 TSO, respectively. Sex, age, treatment group and values for CDAI at baseline and at week 12 visit were kindly made available by Dr. Ralph Mueller (Dr. Falk Pharma GmbH, Falk Foundation e.V., Freiburg, Germany).

\section{NOD2 Genotyping}

Genomic DNA was isolated from whole blood using QIAamp DNA Blood Mini Kit (Qiagen, Hilden, Germany). Genotyping of genomic DNA for 2104C > T (rs2066844), 2722G > C (rs2066845), and 3016_3017insC (rs2066847) was performed using TaqMan technology (Life Technologies, Foster City, CA, United States), as described previously (Schwab et al., 2003). Genotype analyses of $802 \mathrm{C}>\mathrm{T}$ (rs2066842), 1292C > T (rs104895431), and 2863G > A (rs5743291) was performed using predeveloped TagMan Assays (Life Technologies, Foster City, CA, United States) according to manufacturer's protocol on a 7900 Real-Time PCR System. Results were analyzed by use of the Sequence Detection System (SDS) Software (Life Technologies, Foster City, CA, United States).

\section{Statistical Analysis}

Statistical analyses were performed with R-3.4.1 and additional packages SNPassoc 1.9-2 and haplo.stats 1.7.7 (González et al., 2007; R Core Team, 2017; Sinnwell and Schaid, 2018). The primary endpoint remission $(\mathrm{CDAI}<150$ points at Week 12$)$ and the secondary endpoint response (CDAI drop $\geq 100$ at week 12 compared to baseline) were treated as binary variables (yes/no). Four genotyped patients in whom a CDAI value at week 12 was not available were excluded from the analysis. For each variant, multiple logistic regression analysis was applied to estimate the effect of the variant, the treatment as well as the varianttreatment interaction on the primary or secondary endpoint. Here, rs2066842 [Minor allele frequency $(\mathrm{MAF})=37.9 \%$ ] was considered in the additive genetic model and the other five variants (MAF $<10 \%$ ) in the dominant model. Moreover, type II ANOVA was applied to compute corresponding $P$-values (likelihood ratio test).

Haplotype estimation was based on an expectationmaximization (EM) algorithm ( $\mathrm{R}$ package haplo.stats). The most frequent haplotype was defined as the "base" haplotype and haplotypes with a frequency below $5 \%$ were summarized in the "rare" haplotype. A generalized linear model of the haplo.stats package incorporating treatment as covariate without interaction term was used. Haplotypes were coded in the additive model. All tests were two-sided, $P$-value $\leq 0.05$. Given $P$-values were not adjusted for multiple testing.

\section{RESULTS AND DISCUSSION}

Carriers of NOD2 variants are already known to have an increased susceptibility to ileal Crohn's disease (Hugot et al., 2001; Cleynen et al., 2016), and NOD2 variants have been associated with an altered microbial composition (Kennedy et al., 2018) and with decreased expression of antimicrobial peptides ( $\alpha$-defensins), leading to a compromised antibacterial barrier (Wehkamp et al., 2004). In the recent publication by Ramanan et al. (2016) it was speculated that in the subset of patients carrying NOD2 variants, helminth therapy could 
$\mathbf{A}$

\begin{tabular}{|c|c|c|c|c|c|c|c|}
\hline refSNP ID & $\begin{array}{l}\text { Nucleotide } \\
\text { alteration } \\
\text { (NM_022162.1) }\end{array}$ & $\begin{array}{c}\text { Functional } \\
\text { consequence }\end{array}$ & $\begin{array}{c}\text { Protein alteration } \\
\text { (NP_071445.1) }\end{array}$ & Domain & $\begin{array}{l}\text { Minor Allele } \\
\text { Frequency [\%] }\end{array}$ & $\begin{array}{c}\text { HWE } \\
P \text { value } \\
\text { (x2-test) }\end{array}$ & $\begin{array}{c}\text { Minor allele frequency } \\
\text { in general population } \\
{[\%]^{*}}\end{array}$ \\
\hline 152066842 & $802 C>T$ & missense & Pro268Ser & CARD & 37.8 & 0.02 & 24.7 \\
\hline Is104895431 & $1292 \mathrm{C}>\mathrm{T}$ & $\mathrm{m}$ issense & Ser431Leu & NBD & 0.5 & 1 & 0.1 \\
\hline 152066844 & $2104 \mathrm{C}>\mathrm{T}$ & missense & $\mathrm{Arg} 702 \mathrm{Tm}$ & LRR & 9.9 & 0.43 & 5.1 \\
\hline$\sqrt{s 2066845}$ & $2722 \mathrm{G}>\mathrm{C}$ & missense & Gly908Ang & LRR & 3.4 & 0.20 & 1.0 \\
\hline $1 \mathrm{~s} 5743291$ & $2863 \mathrm{G}>\mathrm{A}$ & missense & Va1955\|e & LRR & 8.0 & 0.12 & 10.5 \\
\hline rs2066847 & 3016_3017insC & fram eshift & Leu1007Profs & LRR & 9.7 & 0.03 & 1.4 \\
\hline
\end{tabular}

B

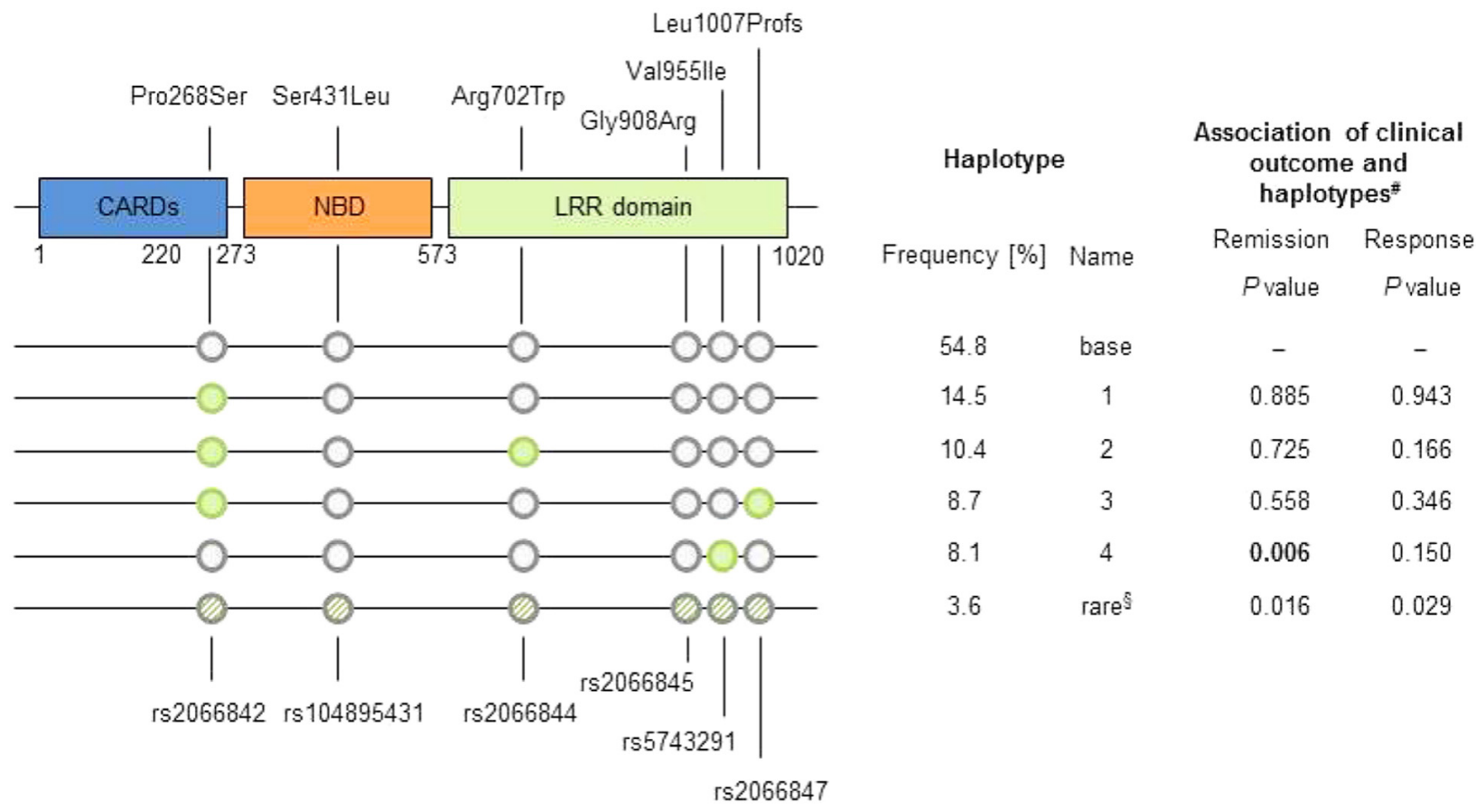

FIGURE 1 | (A) Minor allele frequency in the cohort and general population, and $P$-value from $\chi^{2}$-test of Hardy-Weinberg equilibrium (HWE). *Data from 1000 Genomes Project (Europeans, Phase 3, build 144, http://www.internationalgenome.org/data). (B) Top: Schematic representation of the NOD2 protein, location of changes in amino acid sequence. LRR, leucine rich repeat; CARD, caspase recruitment domain; NBD, nuclear binding domain. Bottom: Graphic representation of haplotypes, sorted by frequency, as well as tabular summary of haplotype analysis result. §"Rare" haplotype summarizes all uncommon haplotypes (frequency < 5\%). "Based on logistic regression analysis comparing each haplotype versus the effect of the "base" haplotype (the most common haplotype).

be more efficacious than in wild-type individuals. Therefore, we analyzed the impact of six important NOD2 variants as a potential important confounder in the TSO trial, which evaluated outcome after 12 weeks of therapy with T. suis ova in Crohn's disease.

We considered the most investigated functional variants Arg702Trp (rs2066844), Gly908Arg (rs2066845) and the frameshift variant Leu1007Profs (rs2066847) that leads to a truncated version of NOD2. In addition, three further coding variants were included in the analysis: the rare variant Ser431Leu (rs104895431) that does not activate NFKB in response to muramyl-dipeptide (Rivas et al., 2011), and the common variants Pro268Ser (rs2066842), with a MAF of 24.7\%, and Val955Ile (rs5743291) with a MAF of $10.5 \%$ in Europeans (see Figure 1A).

The minor allele frequencies of the variants rs2066844, rs2066845, and rs2066847 in our Crohn's disease cohort where higher compared to the general population, similarly as described in the literature (Hugot et al., 2001). The common variant rs2066842 and the frameshift variant rs2066847 slightly deviate from Hardy-Weinberg equilibrium, which is explained as NOD2 is a known susceptibility locus for Crohn's disease.

The influence of the treatment or the genetic variants on the primary endpoint of remission [defined as Crohn's disease activity index (CDAI) $<150$ points after 12 weeks] or the secondary endpoint of response (defined as $\geq 100$ points drop in CDAI after 12 weeks compared to baseline) were evaluated with a multiple logistic regression analysis. No consistent significant association of both outcome measures with the six NOD2 variants or the treatment was observed: remission was significantly associated with the presence of rs5743291, but this could not be ascertained for response, which is clinically implausible. The effect of this variant was 
TABLE 1 | Prevalence of variants in treatment subgroups and logistic regression analyses for two clinical endpoints.

\begin{tabular}{|c|c|c|c|c|c|c|c|}
\hline \multirow[t]{2}{*}{ Genetic variant } & \multirow[t]{2}{*}{ Genotype } & \multirow[t]{2}{*}{ Treatment } & \multirow{2}{*}{$\begin{array}{l}n \text { Remission or } \\
\text { response/total (\%) }\end{array}$} & \multicolumn{4}{|c|}{$P$-values* } \\
\hline & & & & \multicolumn{2}{|c|}{ Remission } & \multicolumn{2}{|c|}{ Response } \\
\hline \multirow[t]{6}{*}{ rs2066842 (Pro268Ser) } & $\mathrm{C} / \mathrm{C}$ & 250 & 7/16 (44) & 0.77 & 0.33 & 0.62 & 0.57 \\
\hline & & placebo & 8/19 (42) & & & & \\
\hline & $\mathrm{C} / \mathrm{T}$ & 250 & $2 / 10(20)$ & & & & \\
\hline & & 2500 & 7/22 (32) & & & & \\
\hline & & 7500 & 8/21 (38) & & & & \\
\hline & & placebo & 15/26 (58) & & & & \\
\hline \multirow[t]{8}{*}{ rs104895431 (Ser431Leu) } & $\mathrm{C} / \mathrm{C}$ & 250 & $13 / 31(42)$ & 0.073 & 1 & 0.082 & 1 \\
\hline & & 2500 & 22/60 (37) & & & & \\
\hline & & 7500 & $24 / 48(50)$ & & & & \\
\hline & & placebo & $28 / 56(50)$ & & & & \\
\hline & $\mathrm{C} / \mathrm{T}$ & 250 & $-^{\#}$ & & & & \\
\hline & & 2500 & $1 / 1(100)$ & & & & \\
\hline & & 7500 & $1 / 1(100)$ & & & & \\
\hline & & placebo & -\# & & & & \\
\hline \multirow[t]{5}{*}{ rs2066844 (Arg702Trp) } & $\mathrm{C} / \mathrm{C}$ & 250 & $11 / 25(44)$ & 0.43 & 0.12 & 0.46 & 0.71 \\
\hline & & 2500 & $19 / 48(40)$ & & & & \\
\hline & & 7500 & $23 / 40(58)$ & & & & \\
\hline & & 7500 & $-^{\#}$ & & & & \\
\hline & & placebo & $1 / 1(100)$ & & & & \\
\hline \multirow[t]{12}{*}{ rs2066845 (Gly908Arg) } & $\mathrm{G} / \mathrm{G}$ & 250 & $11 / 29(38)$ & 0.057 & 0.14 & 0.077 & 0.17 \\
\hline & & 2500 & 20/57 (35) & & & & \\
\hline & & 7500 & 24/46 (52) & & & & \\
\hline & & placebo & $26 / 54(48)$ & & & & \\
\hline & $\mathrm{G} / \mathrm{C}$ & 250 & $2 / 2(100)$ & & & & \\
\hline & & 2500 & $3 / 4(75)$ & & & & \\
\hline & & 7500 & 1/3 (33) & & & & \\
\hline & & placebo & $1 / 1(100)$ & & & & \\
\hline & $\mathrm{C} / \mathrm{C}$ & 250 & $-{ }^{\#}$ & & & & \\
\hline & & 2500 & $-\#$ & & & & \\
\hline & & 7500 & -\# & & & & \\
\hline & & placebo & $1 / 1(100)$ & & & & \\
\hline \multirow[t]{4}{*}{ rs5743291 (Val955lle) } & $\mathrm{G} / \mathrm{G}$ & 250 & 8/24 (33) & 0.0051 & 0.92 & 0.35 & 0.55 \\
\hline & & 2500 & $18 / 52(35)$ & & & & \\
\hline & & 7500 & $18 / 40(45)$ & & & & \\
\hline & & placebo & $25 / 52(48)$ & & & & \\
\hline
\end{tabular}


TABLE 1 | Continued

\begin{tabular}{|c|c|c|c|c|c|c|c|}
\hline \multirow[t]{3}{*}{ Genetic variant } & \multirow[t]{3}{*}{ Genotype } & \multirow[t]{3}{*}{ Treatment } & \multirow{3}{*}{$\begin{array}{l}n \text { Remission or } \\
\text { response/total (\%) }\end{array}$} & \multicolumn{4}{|c|}{$P$-values* } \\
\hline & & & & \multicolumn{2}{|c|}{ Remission } & \multicolumn{2}{|c|}{ Response } \\
\hline & & & & Main effect & $\begin{array}{l}\text { Variant-treatment } \\
\text { interaction effect }\end{array}$ & Main effect & $\begin{array}{l}\text { Variant-treatment } \\
\text { interaction effect }\end{array}$ \\
\hline & $A / G$ & 250 & $5 / 7(71)$ & & & & \\
\hline & & 2500 & $3 / 6(50)$ & & & & \\
\hline & & 7500 & $7 / 9(78)$ & & & & \\
\hline & & placebo & $3 / 4(75)$ & & & & \\
\hline & $\mathrm{A} / \mathrm{A}$ & 250 & -\# & & & & \\
\hline & & 2500 & $2 / 3(67)$ & & & & \\
\hline & & 7500 & $-\#$ & & & & \\
\hline & & placebo & $-^{\#}$ & & & & \\
\hline \multirow[t]{12}{*}{ rs2066847 (Leu1007Profs) } & $-1-$ & 250 & $11 / 28(39)$ & 0.69 & 0.23 & 0.3 & 0.49 \\
\hline & & 2500 & 19/50 (38) & & & & \\
\hline & & 7500 & $22 / 45(49)$ & & & & \\
\hline & & placebo & $24 / 43(56)$ & & & & \\
\hline & -/insC & 250 & 2/3 (67) & & & & \\
\hline & & 2500 & $4 / 11(36)$ & & & & \\
\hline & & 7500 & $2 / 3(67)$ & & & & \\
\hline & & placebo & 4/10 (40) & & & & \\
\hline & insC/insC & 250 & $-\#$ & & & & \\
\hline & & 2500 & -\# & & & & \\
\hline & & 7500 & $1 / 1(100)$ & & & & \\
\hline & & placebo & 0/3 (0) & & & & \\
\hline
\end{tabular}

*Unadjusted P-value based on multiple logistic regression and type II ANOVA. \#No patients with corresponding variant-treatment combination in cohort.

independent of the treatment group. No significant interaction effect between the variants and any treatment group was evident (Table 1).

We further computed haplotypes in the cohort from the unphased genotype data. A pre-dominating haplotype ("base" haplotype) was found in $54.8 \%$ of cases, and additional four haplotypes showed a frequency of $8 \%$ or more (Figure 1B). The haplotype 4 differs from the "base" haplotype solely in rs5743291 and shows the same inconsistent association to the binary clinical endpoints as rs5743291 in the single variant analysis described above. Considering the study endpoints as continuous variables (i.e., CDAI count at follow-up or difference between CDAI count at follow-up and CDAI count at baseline) did not reveal any additional significant associations after correction for multiple testing.

Interestingly, the two patients who carried the rare functional variant rs104895431 had been randomized to the verum group and showed a remarkable benefit from treatment, i.e., a decrease in CDAI from 245 to 127 (patient received 2500 TSO), and from 346 to 60 points (7500 TSO). The latter patient simultaneously carried two further variants, rs2066842 and the frameshift variant rs2066847. Although this might be due to pure coincidence, the response to TSO treatment in the individual carrying three functional variants is noteworthy.

Yet in general, NOD2 variants seem not to have a clinically meaningful impact on the outcome of the TSO trial, which can be explained by various reasons.
Firstly, as Nelson et al. (2016) have outlined, only rarely, even truly efficacious drugs will have a clinically meaningful genetic predictor of efficacy. In general, surrogate endpoints, e.g., a decrease in serum CRP, could help detecting differences in subgroups, but there was no overall response in terms of laboratory markers of intestinal inflammation in the original trial. Only blood eosinophilic counts, eosinophil derived neurotoxin in stools and the T. suis E/S antigen specific IgG showed dosedependent effects (Schölmerich et al., 2017).

Secondly, the randomization process of the clinical study did not stratify according to genotype. That means, genetic variants and treatments are haphazardly distributed, e.g., heterozygous Leu1007Profs variants were present in $17 \%$ of placebo-treated individuals, but only in $8 \%$ of patients treated with 7500 T. suis ova.

Further, it has to be taken into account that the mostly heterozygous carriers of variants in the NOD2 gene encountered in the TSO trial cannot be easily compared to a complete NOD2 knock-out. Even in the experiments done with Nod2-/mice by Bowcutt et al. (2014) which demonstrated the importance of Nod2 in dentritic cell recruitment and early phases of the immune response, the knock-outs were ultimately able to expel $T$. muris. It would be interesting to know if specific variant models yielded similar effects in the animal model. The Arg702Trp, Gly908Arg, Val955Ile, and the frameshift variants affect the leucine-rich repeat domain of the NOD2 protein and hamper bacterial recognition, but may 
not abbrogate all functions of NOD2 relevant to intestinal inflammation.

The impact of NOD2 on microbial composition itself is still controversially discussed. For example, if $\mathrm{Nod} 2^{-/-}$and wild-type mice were co-housed, a Nod2 genotype-specific effect on gut microbiota could no longer be demonstrated (Shanahan et al., 2014) - although coprophagy may have been a confounding variable. Other investigations have even shown that diet might be more relevant than genetic host factors (Carmody et al., 2015). To the contrast, Al Nabhani et al. (2016) did report Nod2-related differences in mucosal microbiota employing an embryo transfer strategy, which helped minimizing environmental and maternal influences.

Obviously, findings from in vivo mouse experiments and human pathophysiology might not always be in line, and T. suis adds even more complexity to this already intricate interaction. To conclude, although analyzing the genetic make-up of the well-characterized study population in this randomized trial was tempting, clinical efficacy of $T$. suis treatment was not better in carriers of six relevant NOD2 variants.

\section{ETHICS STATEMENT}

This study was carried out in accordance with the International Conference on Harmonisation (ICH) Guideline for Good Clinical Practice and was approved by independent ethic committees for each study center. All subjects gave written informed consent for original clinical trial and all patients included in this additional genetic analysis have signed a separate, optional informed consent.

\section{REFERENCES}

Al Nabhani, Z., Lepage, P., Mauny, P., Montcuquet, N., Roy, M., Le Roux, K., et al. (2016). Nod2 deficiency leads to a specific and transmissible mucosaassociated microbial dysbiosis which is independent of the mucosal barrier defect. J. Crohns Colitis 10, 1428-1436. doi: 10.1093/ecco-jcc/jjw095

Bowcutt, R., Bramhall, M., Logunova, L., Wilson, J., Booth, C., Carding, S. R., et al. (2014). A role for the pattern recognition receptor Nod2 in promoting recruitment of $\mathrm{CD} 103++$ dendritic cells to the colon in response to Trichuris muris infection. Mucosal Immunol. 7, 1094-1105. doi: 10.1038/mi.2013.125

Carmody, R. N., Gerber, G. K., Luevano, J. M., Gatti, D. M., Somes, L., Svenson, K. L., et al. (2015). Diet dominates host genotype in shaping the murine gut microbiota. Cell Host Microbe 17, 72-84. doi: 10.1016/j.chom.2014.11.010

Cleynen, I., Boucher, G., Jostins, L., Schumm, L. P., Zeissig, S., Ahmad, T., et al. (2016). Inherited determinants of Crohn's disease and ulcerative colitis phenotypes: a genetic association study. Lancet 387, 156-167. doi: 10.1016/ S0140-6736(15)00465-1

González, J. R., Armengol, L., Solé, X., Guinó, E., Mercader, J. M., Estivill, X., et al. (2007). SNPassoc: an R package to perform whole genome association studies. Bioinformatics 23, 644-645. doi: 10.1093/bioinformatics/btm025

Hiemstra, I. H., Klaver, E. J., Vrijland, K., Kringel, H., Andreasen, A., Bouma, G., et al. (2014). Excreted/secreted Trichuris suis products reduce barrier function and suppress inflammatory cytokine production of intestinal epithelial cells. Mol. Immunol. 60, 1-7. doi: 10.1016/j.molimm.2014.03.003

Hugot, J. P., Chamaillard, M., Zouali, H., Lesage, S., Cézard, J. P., Belaiche, J., et al. (2001). Association of NOD2 leucine-rich repeat variants with susceptibility to Crohn's disease. Nature 411, 599-603. doi: 10.1038/35079107

\section{AUTHOR CONTRIBUTIONS}

SJ drafted the manuscript, organized and acquired data, took part in statistical analysis, interpreted data and was clinical investigator in original TSU trial. ES coordinated the genetic analysis, interpreted the data and critically revised the manuscript. SW and RT are biostatisticians who performed the statistical analysis and critically revised the manuscript. JS was the lead investigator of the original clinical trial and critically revised the manuscript. NM provided reagents and provided comments on drafts. EFS was investigator in the original clinical trial and critically revised the manuscript. MS contributed to conception and design of the study, provided funding and critically revised the manuscript. JW was principal investigator in the clinical trial, contributed to conception and design of the study, provided funding and critically revised the manuscript.

\section{FUNDING}

The work was supported by the Robert Bosch Stiftung, Stuttgart, Germany. JW is supported by the Heisenberg Program of the Deutsche Forschungsgemeinschaft. JW holds a grant from Deutsche Forschungsgemeinschaft (DFG) with the project number 238358106.

\section{ACKNOWLEDGMENTS}

We thank Ralph Mueller from Falk Pharma, who was the principal scientific investigator of the original trial and made available the clinical data for correlation in this study. We thank Andrea Jarmuth for her excellent technical assistance.

Kennedy, N. A., Lamb, C. A., Berry, S. H., Walker, A. W., Mansfield, J., Parkes, M., et al. (2018). The impact of NOD2 variants on fecal microbiota in Crohn's disease and controls without Gastrointestinal disease. Inflamm. Bowel Dis. 24, 583-592. doi: 10.1093/ibd/izx061

Nelson, M. R., Johnson, T., Warren, L., Hughes, A. R., Chissoe, S. L., $\mathrm{Xu}$, C.-F., et al. (2016). The genetics of drug efficacy: opportunities and challenges. Nat. Rev. Genet. 17, 197-206. doi: 10.1038/nrg. 2016.12

R Core Team (2017). A Language and Environment for Statistical Computing. Vienna: R Foundation for Statistical Computing.

Ramanan, D., Bowcutt, R., Lee, S. C., Tang, M. S., Kurtz, Z. D., Ding, Y., et al. (2016). Helminth infection promotes colonization resistance via type 2 immunity. Science 352, 608-612. doi: 10.1126/science.aaf 3229

Ramanan, D., Tang, M. S., Bowcutt, R., Loke, P., and Cadwell, K. (2014). Bacterial sensor Nod2 prevents inflammation of the small intestine by restricting the expansion of the commensal Bacteroides vulgatus. Immunity 41, 311-324. doi: 10.1016/j.immuni.2014.06.015

Rivas, M. A., Beaudoin, M., Gardet, A., Stevens, C., Sharma, Y., Zhang, C. K., et al. (2011). Deep resequencing of GWAS loci identifies independent rare variants associated with inflammatory bowel disease. Nat. Genet. 43, 1066-1073. doi: 10.1038/ng.952

Sandborn, W. J., Elliott, D. E., Weinstock, J., Summers, R. W., LandryWheeler, A., Silver, N., et al. (2013). Randomised clinical trial: the safety and tolerability of Trichuris suis ova in patients with Crohn's disease. Aliment. Pharmacol. Ther. 38, 255-263. doi: 10.1111/apt. 12366 
Schölmerich, J., Fellermann, K., Seibold, F. W., Rogler, G., Langhorst, J., Howaldt, S., et al. (2017). A randomised, double-blind, placebo-controlled trial of Trichuris suis ova in active Crohn's disease. J. Crohns Colitis 11, 390-399. doi: 10.1093/ecco-jcc/jjw184

Schwab, M., Schaeffeler, E., Marx, C., Fromm, M. F., Kaskas, B., Metzler, J., et al. (2003). Association between the C3435T MDR1 gene polymorphism and susceptibility for ulcerative colitis. Gastroenterology 124, 26-33. doi: 10.1053/ gast.2003.50010

Shanahan, M. T., Carroll, I. M., Grossniklaus, E., White, A., von Furstenberg, R. J., Barner, R., et al. (2014). Mouse Paneth cell antimicrobial function is independent of Nod2. Gut 63, 903-910. doi: 10.1136/gutjnl-2012304190

Sinnwell, J. P., and Schaid, D. J. (2018). haplo.stats: Statistical Analysis of Haplotypes with Traits and Covariates when Linkage Phase is Ambiguous. R Package Version 1.7.7. Avaialable at: https://cran.r-project.org/web/packages/haplo.stats/index. html

Summers, R. W., Elliott, D. E., Urban, J. F., Thompson, R., and Weinstock, J. V. (2005a). Trichuris suis therapy in Crohn's disease. Gut 54, 87-90. doi: 10.1136/ gut.2004.041749
Summers, R. W., Elliott, D. E., Urban, J. F., Thompson, R. A., and Weinstock, J. V. (2005b). Trichuris suis therapy for active ulcerative colitis: a randomized controlled trial. Gastroenterology 128, 825-832.

Wehkamp, J., Harder, J., Weichenthal, M., Schwab, M., Schäffeler, E., Schlee, M., et al. (2004). NOD2 (CARD15) mutations in Crohn's disease are associated with diminished mucosal alpha-defensin expression. Gut 53, 1658-1664. doi: 10.1136/gut.2003.032805

Conflict of Interest Statement: The authors declare that the research was conducted in the absence of any commercial or financial relationships that could be construed as a potential conflict of interest.

Copyright (C) 2018 Jaeger, Schaeffeler, Winter, Tremmel, Schölmerich, Malek, Stange, Schwab and Wehkamp. This is an open-access article distributed under the terms of the Creative Commons Attribution License (CC BY). The use, distribution or reproduction in other forums is permitted, provided the original author(s) and the copyright owner(s) are credited and that the original publication in this journal is cited, in accordance with accepted academic practice. No use, distribution or reproduction is permitted which does not comply with these terms. 